\title{
Development of a scintillation counter with MPPC readout for the internal tagging system
}

\author{
Hiroki KANDA*, Yuma KASAI, Kazushige MAEDA, Takashi NISHIZAWA, and \\ Fumiya YAMAMOTO \\ Department of Physics, Tohoku University, Sendai 980-8578, Japan \\ E-mail: kanda@m.tohoku.ac.jp
}

\begin{abstract}
We have been developing an array of scintillation counters as an electron detector for a photon tagging spectrometer at the Research Center for Electron Photon Science, Tohoku University. Since it is installed in the limited space of the bending magnet of the accelerator, a compact size and tolerance to the magnetic field are required. We also aim at the improvement of the timing resolution of the counter in order to set a strict event selection. For the photosensor of the scintillation counter, we chose the multi-pixel photon counter (MPPC) which fulfills those requirements. We made a prototype scintillation counter with a plastic scintillator in a shape of a small rectangular prism directly coupled to the MPPC. We developed a dedicated compact amplifier circuit board on which two MPPC's and two sets of amplifier circuits are mounted. We fabricated a prototype of the electron detector of the photon tagging system with four scintillation counters with MPPC readouts. We performed a test with the electrons and positrons converted from a high energy photon beam. We selected single-electron-passing events and measured their coincidence timing. The timing resolutions of the counters were obtained from the fluctuations of the coincidence timing. The best intrinsic timing resolution of the counter was $80 \mathrm{ps}$ (RMS). The best timing resolutions of all four scintillation counters ranged from $80 \mathrm{ps}$ to $110 \mathrm{ps}$. The timing resolution and the range of their indivisual difference are appropriate for the use as the electron detector for the photon tagging system.
\end{abstract}

International Workshop on New Photon-detectors

June 13-15, 2012

LAL Orsay, France

\footnotetext{
* Speaker.
} 


\section{The tagged photon beam at ELPH}

The meson photoproduction on the nucleon has been investigated for the exploration of the nucleon resonances, the photon absorption mechanism, and the exotic states of the baryons. We have been investigating the kaon photoproduction[1,2] and the double pion photoproduction[3, $4,5]$ on the deuteron with the Neutral Kaon Spectrometer-2 (NKS2) at the Research Center for Electron Photon Science, Tohoku University (ELPH). We aim at the investigation of the neutral kaon photoproduction and the double pion photoproduction with $1 \mathrm{GeV}$ region photon beams.

The Stretcher and Booster Ring (STB), which accelerates the electrons up to $1.2 \mathrm{GeV}$, has been operational at ELPH. An internal photon tagging system, the STB Tagger, is installed in it[6] for providing the photon beams to the NKS2. It utilizes a movable radiator to produce bremsstrahlung photons and a set of scintillation counters to measure the number and the momenta of post-bremsstrahlung electrons. Figure 1 shows the schematic view of the STB Tagger. One of the bending magnet of STB is used for the momentum analysis of the post-bremsstrahlung electrons. A set of scintillation counters is installed in the space surrounded by the poles, the coils and the yoke of the magnet. It consists of 50 finger counters (TagF) and 12 backup counters (TagB). These counters are aligned to the orbit of the post-bremsstrahlung electrons. One TagB counter covers the electron orbits which pass through four TagF counters. By using the coincidence between TagF and $\mathrm{TagB}$, we can select the orbit of the electron to reduce the background hits and measure the momentum of the electron. The measured momentum of an electron is directly translated into its energy $E^{\prime}$. By using $E^{\prime}$ and the energy of the circulating electron $\left(E_{0}\right)$, the energy of the photon $\left(E_{\gamma}\right)$ is obtained with a formula: $E_{\gamma}=E_{0}-E^{\prime}$. The energy range of the tagged photon is from $\frac{2}{3} E_{0}$ to $\frac{11}{12} E_{0}$. The TagB counter provides the precise timing of the passing of the electron as well. The timing information is used for the coincidence with the NKS2 detectors to reduce the background and set a strict event selection.

The STB and the STB Tagger were affected by the huge earthquake on 11 Mar. 2011. We started the recovery and upgrade of the tagger. The properties of the STB Tagger and the goal of the upgraded tagger is summarized in Table 1. The important point in the design of the electron detector of the tagging system is that they have to be installed in the narrow space inside the bending magnet where the magnetic flux density ranges from 0.3 to $0.7 \mathrm{~T}$. In order to locate the scintillation counters in these environment, the STB Tagger utilized a light guide with a bundle of long optical fibers to place the photomultiplier tubes (PMT's) out of the magnet. However, the light guides reduced the photoelectron yield and increased the timing spread. The typical timing resolution of the TagB counter was 350 ps (RMS). In the upgraded photon tagger, we plan to use Hamamatsu Multi-pixel Photon Counter (MPPC) [7] for the photo-sensor of the scintillation counter. It has the following characteristics which are suitable for this purpose, (1) a small size (an aperture of $3 \times 3 \mathrm{~mm}^{2}$ ) that matches the small scintillator, (2) tolerance to the magnetic field, and (3) a good timing resolution (500 - 600 in FWHM).

\section{Development of the scintillation counter}

Though the nominal timing resolution of the MPPC fulfills our requirement, the timing resolutions of the scintillation counters using the MPPC's varies in their applications. A timing resolution 


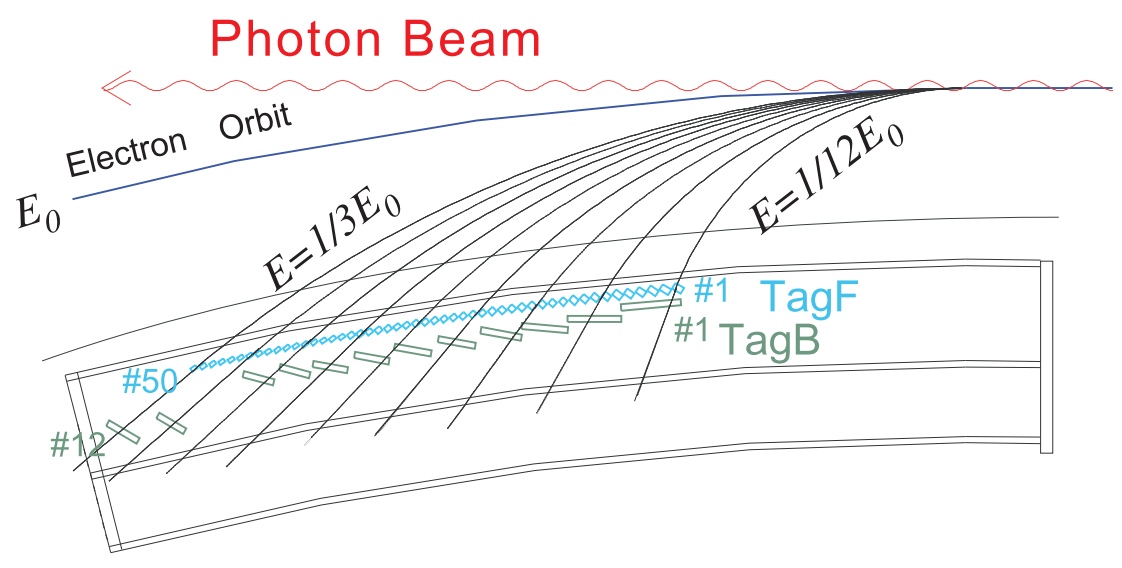

Figure 1: Schematic view of the STB Tagger. Two kinds of counters, TagF's and TagB's are aligned in two rows for the detection of deflected electrons.

Table 1: Properties of the STB Tagger and the goal of the upgraded tagger

\begin{tabular}{lccl}
\hline Item & Current system & Goal & Comment \\
\hline Total energy range & $300 \mathrm{MeV}$ & $350 \mathrm{MeV}$ & $E_{\gamma \max } \sim 1.15 \mathrm{GeV}$ for $E_{0}=1.2 \mathrm{GeV}$ \\
Mean energy span & $6 \mathrm{MeV}$ & $3 \mathrm{MeV}$ & \\
Number of TagF counters & 50 & 120 & \\
Widths of TagF counters & $3-8 \mathrm{~mm}$ & $3 \mathrm{~mm}$ & \\
Timing resolution (RMS) & $350 \mathrm{ps}$ & $<200 \mathrm{ps}$ & Comparable to other TOF counters \\
\hline
\end{tabular}

of a polystyrene-based slab scintillator counter with fiber/MPPC readout was reported as $1 \mathrm{~ns}$ and it was dependent on the light yield [9]. LYSO counters for time-of-flight PET detectors with high gain amplifiers showed an average timing resolution of $\sim 310$ ps (FWHM) [10]. For taking full advantage of the good timing property of the MPPC, we focused on maximizing the light yield and optimizing the amplifier.

There are three kinds of pixel sizes of the MPPC, namely $25 \mu \mathrm{m}, 50 \mu \mathrm{m}$, and $100 \mu \mathrm{m}$. The detection efficiency and gain of the MPPC correlate with the size of the pixel. We selected the MPPC with $100 \mu \mathrm{m}$ pixel for maximizing the gain and efficiency. We estimated the light yields with the emission spectra of the plastic scintillator supplied by ELJEN Technology [11] and the spectral detection efficiency of the MPPC. The maximum light yield was obtained with the ELJEN EJ-204 scintillator. Thanks to its small size and tolerance to the magnetic field, the MPPC can be directly coupled to the scintillator and located in the bending magnet. Thus, we can reduce the transmission loss of the scintillation photon.

We developed a dedicated amplifier circuit taking advantage of a broad bandwidth and a high slew rate of an ultrahigh speed operational amplifier, AD8000 supplied by Analog Devices [12]. The amplifier is an inverting current-to-voltage converter as shown in the schematic diagram in Fig. 2. The amplifier circuit is coupled to the MPPC with a capacitor with a small capacitance (47 $\mathrm{pF})$. It suppresses a fluctuation of a base line originated from a dark current and amplifies a high frequency component of the signal for higher timing resolution. Two MPPC's and two circuits of the amplifiers are mounted on both sides of a compact printed circuit board (PCB) with the size 

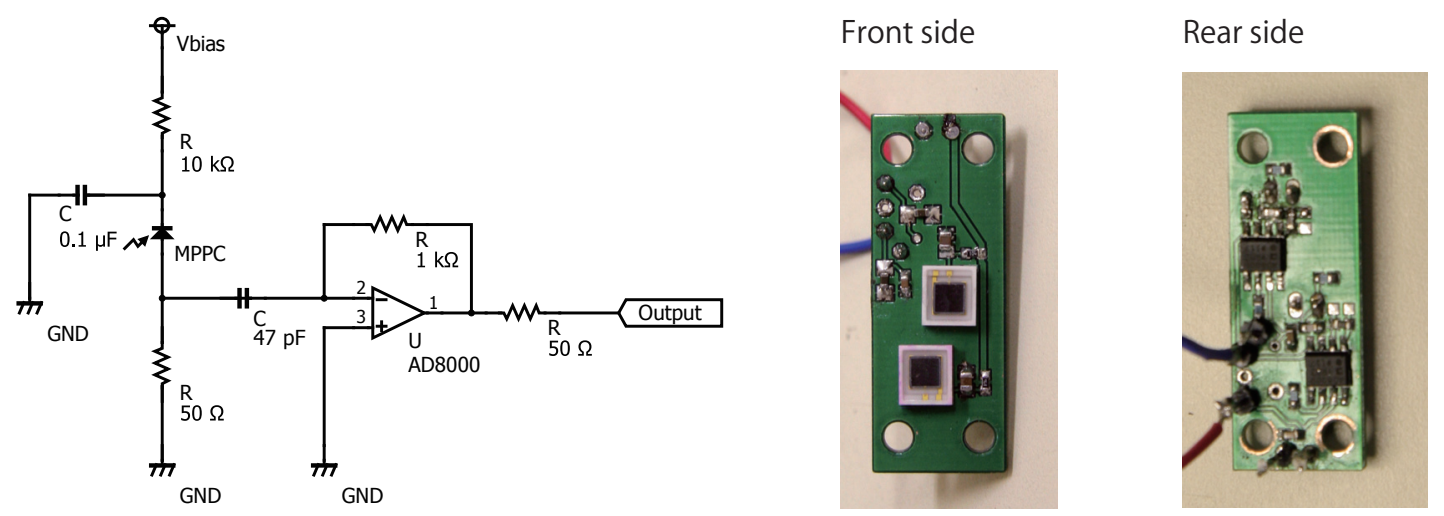

Figure 2: The schematic diagram of the amplifier circuit (left) and the photographs of the amplifier circuit board with two circuits (center for the front side and right for the rear side).

of $15 \times 40 \mathrm{~mm}^{2}$ as shown in the photographs in Fig. 2. The timing resolution of the MPPC and the amplifier circuit was tested with an LED pulser which simulates the emission time profile of the plastic scintillator. The obtained timing resolution ranged from $80 \mathrm{ps}$ to 110 ps with the bias voltage from 0.24 to $7.24 \mathrm{~V}$ over the operating voltage in the data sheets.

We assembled a prototype detector with four Finger counters and one Backup counter which simulates one combination of 4 TagF's and 1 TagB. A plastic scintillator with a size of $3 \times 3 \times$ $20 \mathrm{~mm}^{3}$ was triply wrapped with a Teflon tape and coupled on the aperture of the MPPC. A 1$\mathrm{mm}$ thick silicone rubber sheet, EJ-560 [11], was inserted between the scintillator prism and the aperture of the MPPC to ensure the tight optical contact and to avoid the damage on the aperture due to the stress to push the scintillator against it. Four small scintillation counters with MPPC readout and a scintillation counter with PMT readout were placed in a small aluminum case. The small scintillation counters with the MPPC's were named as the Finger-1 to Finger-4 and the other one with the PMT as the Backup counter. We used a fine-mesh PMT (Hamamatsu H6152-01B) which are operational in a strong magnetic field which can be a candidate for the photon sensor for TagB counter in the new tagger. By designing and assembling the prototype for the test of the performance of the scintillation counters, we also learned the policy and the method of the fabrication of the tagger.

\section{Test with a photon beam}

We performed a test experiment of the prototype at NewSUBARU. It is the electron storage ring and provides a few tens of $\mathrm{MeV}$ photon beam at the beam line 1 by the laser Compton backscattering $[13,14]$. The top energy of the photon is $32.7 \mathrm{MeV}$ at the setting of the electron energy of $974 \mathrm{MeV}$ and the wave length of the laser of $532 \mathrm{~nm}$. Figure 3 shows the setup of the detectors. We placed one more scintillation counter named as Reference counter with the same construction with the Backup counter. We placed a lead collimator with a 3-mm-diameter hole, a $2 \mathrm{~mm}$-thick lead plate as the converter, the Reference counter and the prototype detector along the beam line. The irradiated photons on the lead plate were converted to the electrons and positrons via the pair-creation process, which passed through these scintillation counters. We set a trigger with 


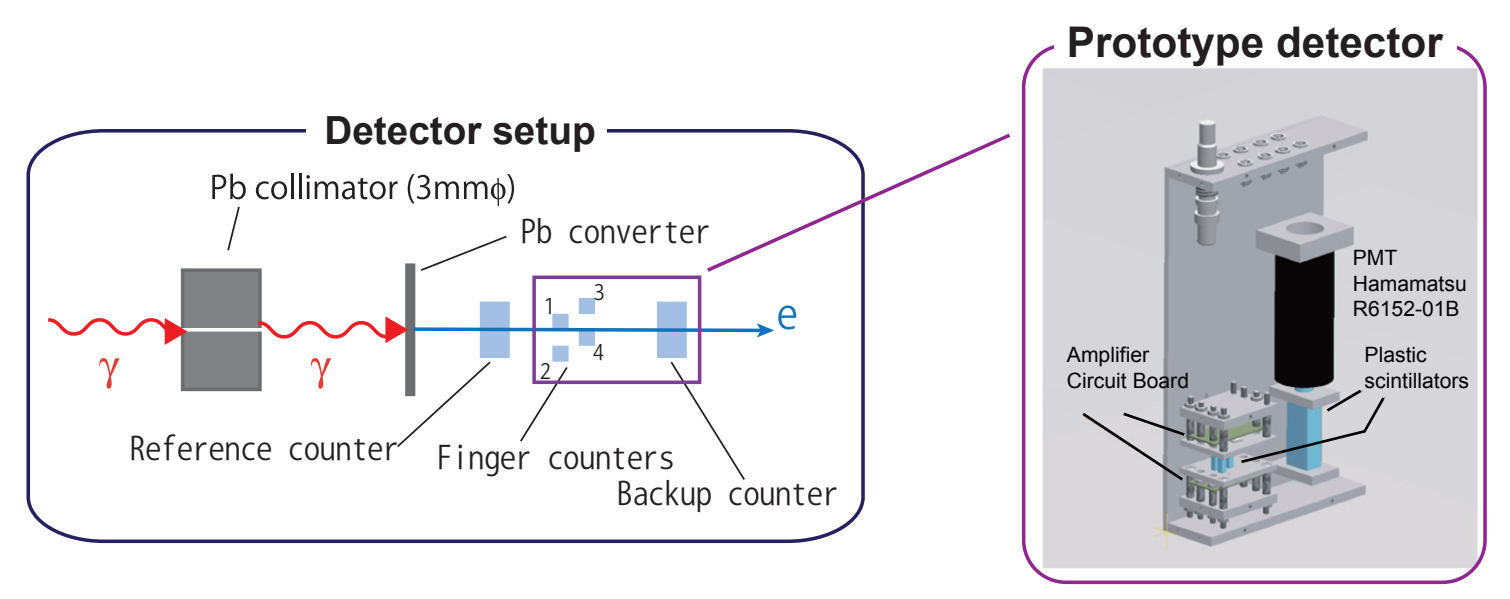

Figure 3: The setup of the prototype detector on the beam line.

the coincidence of the reference counter and the backup counter to acquire data with a CAMAC charge ADC and a CAMAC high resolution TDC ( 25 ps per channel). We selected $\pm 2 \sigma$ regions of the single electron passing peaks in the ADC spectra for the reference, the Finger and the backup counter after fitting the Landau distribution or the Gaussian function according to the shapes of their ADC spectra. We performed a slewing correction for the compensation of the time walk. By measuring the coincidence timings between three combinations of three counters and solving the simultaneous equations, we derived the timing resolution of each counter. The operating voltages in the data sheets for four MPPC's was roughly $70.8 \mathrm{~V}$. We scanned the bias voltage for MPPC's of the Finger counters from $70.6 \mathrm{~V}$ to $72.0 \mathrm{~V}$ with an interval of $0.2 \mathrm{~V}$. We set the constant threshold voltage for the discriminators as $30 \mathrm{mV}$ which corresponded to $\sim 20$ photoelectrons at the bias of $71.0 \mathrm{~V}$ for the Finger-1 counter. Its typical number of photoelectrons for a single electron passing was estimated as 400 . The corresponding timing resolution was $120 \mathrm{ps}$ (RMS). The timing resolutions of the four finger counters with respect to the supplied bias voltage are plotted in Fig. 4. The best timing resolution was obtained as $80 \mathrm{ps}$ at the bias of $71.4 \mathrm{~V}$ for the Finger- 1 counter. The best timing resolutions of other Finger counters ranged from 90 to 110 ps at the bias higher than their operating voltages. The timing resolution itself fulfills our requirement. Its small individual deviation is also suitable for our use.

\section{Summary}

We have developed a small scintillation counter with MPPC readout to be used in the internal tagging system at ELPH. It consists of a small plastic scintillator with a size of $3 \times 3 \times 20 \mathrm{~mm}^{3}$, a transparent silicone rubber, an MPPC S10362-33-100C, and a dedicated amplifier. The amplifier was developed utilizing an ultrahigh speed operational amplifier AD8000. We assembled four scintillation counters with MPPC readout and one scintillation counter with PMT readout as a prototype of the detector for the photon tagger. We performed the beam test of the prototype detector at the NewSUBARU BL1, and obtained the best timing resolution of the counter as 80 


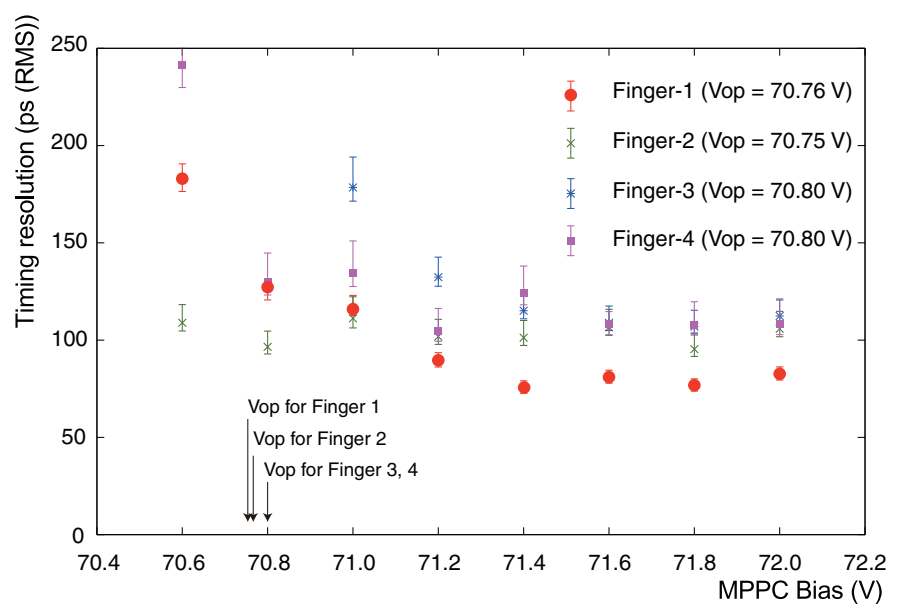

Figure 4: Bias dependence of the timing resolutions of four finger counters.

ps. It fulfills our requirements to the counter for the timing resolution. We continue developing the internal tagger system aiming at the completion in the next fiscal year.

Authors are greatly thankful to the special support by Prof. S. Amano, Dr. K. Horikawa, Prof. S. Miyamoto and all other staffs in NewSUBARU, Laboratory of Advanced Science and Technology for Industry, University of Hyogo. This program was partly supported by JPSP KAKENHI Grant Number 23540334.

\section{References}

[1] M. Kaneta et al., Proceedings of SENDAI08 (2010), 63-70.

[2] K. Futatsukawa et al., EPJ Web of Conferences 20 (2012) 02005.

[3] Y. C. Han et al., Nucl. Phys. A 834 (2010) 596c-598c.

[4] H. Kanda et al., Nucl. Phys. A 835 (2010) 317-320.

[5] H. Kanda et al., EPJ Web of Conferences 20 (2012) 02006.

[6] H. Yamazaki et al., Nucl. Instr. and Meth. A 389 (2005) 10.

[7] Data sheet for S10362-33 series: Cat. No. KAPD1023E05, Hamamatsu K.K. (2009).

[8] Data sheet for S10362-11 series: Cat. No. KAPD1022E05, Hamamatsu K.K. (2009).

[9] A. Izmaylov et al., Nucl. Instr. and Meth. A 623 (2010) 382.

[10] Chang Long Kim et al., IEEE Trans. Nucl. Sci. 58 (2011) 3.

[11] ELJEN Technology, http://www.eljentechnology.com/.

[12] Data sheet for AD8000: No. D05321-0-3/10(A), Analog Devices Inc. (2010).

[13] S. Amano et al. Nucl. Instrum. and Meth. A602 (2009) 337 - 341.

[14] K. Horikawa et al., Nucl. Instrum. and Meth. A618 (2010) 209 - 215. 